\title{
Effect of folic acid supplementation on mood and serotonin response in healthy males
}

\author{
Emma Williams ${ }^{1}$, Barbara Stewart-Knox ${ }^{1}$, Ian Bradbury ${ }^{2}$, Ian Rowland ${ }^{1}$, Kristina Pentieva ${ }^{1}$, Anders Helander ${ }^{3}$ \\ and Helene McNulty ${ }^{1}$ \\ ${ }^{1}$ Northern Ireland Centre for Food and Health (NICHE), University of Ulster, Coleraine BT52 1SA, Northern Ireland \\ ${ }^{2}$ School of Biomedical Sciences, University of Ulster, Coleraine, Northern Ireland \\ ${ }^{3}$ Department of Clinical Neuroscience, Karolinska Institute, Stockholm, Sweden
}

(Received 15 October 2004 - Revised 2 March 2005 - Accepted 7 April 2005)

\begin{abstract}
Evidence suggests that low folate status may be detrimental to mood and associated with depleted cerebrospinal fluid levels of the neurotransmitter serotonin (5-hydroxytryptamine; 5-HT). A placebo-controlled trial was carried out to determine the effect of folic acid supplementation (100 $\mu \mathrm{g}$ for 6 weeks followed by $200 \mu \mathrm{g}$ for a further 6 weeks) upon subjective mood (Positive and Negative Affect Schedule) and biochemical markers of mood (5-HT) in healthy males ( $n$ 23). Blood samples were obtained at baseline (week 0) and during the intervention at week 6 and week 12. Subjective mood assessments were obtained at week 0 and week 12 . The results showed an increase in serum and erythrocyte folate concentrations $(P=0.02$ and $P=0 \cdot 003$, respectively) and a corresponding decrease in plasma homocysteine $(P=0.015)$ in response to the folic acid intervention. Neither subjective mood nor 5-HT levels, however, were significantly altered in response to the change in folate status. Folic acid given at physiological doses did not appear to improve the mood of healthy folatereplete individuals over a 12-week period. Further research is needed to address the effect of folic acid supplementation or of longer duration or increased dose, particularly in the face of sub-optimal folate status.
\end{abstract}

Folate: Folic acid: Mood: Positive and Negative Affect Schedule: Serotonin: Homocysteine

More than 40 years ago, Victor Herbert (1962) consumed a folatedeficient diet $(5 \mu \mathrm{g} / \mathrm{d})$, as a result of which he experienced symptoms of insomnia, irritability, fatigue and forgetfulness. These symptoms disappeared in response to low-dose folic acid $(250 \mu \mathrm{g} / \mathrm{d})$. Since then, the importance of folate for psychological well-being has become increasingly recognised. Among psychiatric patients with depression, up to a third have been found to have low folate status (Carney, 1967; Reynolds et al. 1970). There is also some evidence to suggest that intervention with folic acid can improve mood in those with depression (Botez et al. 1979a; Guaraldi et al. 1993). Lower serum and erythrocyte folate concentrations have been found to be associated with increased severity in the symptoms of depression (Carney, 1967; Ghadirian et al. 1980; Wesson et al. 1994). Response to antidepressant medication has also been found to be dependent upon folate status (Reynolds et al. 1970; Wesson et al. 1994; Fava et al. 1997) and enhanced by folic acid supplementation (Coppen et al. 1986; Godfrey et al. 1990; Coppen \& Bailey, 2000). There is therefore accumulating evidence to suggest that folate is psychoactive and that folic acid supplementation may bring about psychological benefit.

With regard to the potential link between folate and depression, Reynolds \& Carney (1984) put forward the 'methylation hypothesis of depression', whereby methylation processes in the nervous system are thought to influence mood. Methylation is dependent upon levels of the major methyl donor $S$-adenosylmethionine (SAM), which is derived from the methionine cycle. During this cycle, folate in the form 5-methyltetrahydrofolate is required to convert homocysteine to L-methionine. Methionine then combines with ATP to form SAM, which in turn is required for many transmethylation reactions (up to thirty-five) in the central nervous system (Cantoni, 1953; Bottiglieri et al. 1994). Low folate status/intake is known to lead to increased homocysteine concentrations (Brattstrom et al. 1998), which in turn are associated with lower levels of methionine and ultimately lower SAM. This may have implications for mood. SAM is considered important in the regulation of mood, as it has been shown to increase cerebrospinal fluid levels of 5-hydroxyindoleacetic acid in those with depression (Ordonez \& Wurtman, 1974; Agnoli et al. 1977; Bottiglieri et al. 1994). 5-Hydroxyindoleacetic acid is the main metabolite for the monoamine neurotransmitter serotonin (5-hydroxytryptamine; 5-HT). Decreased synthesis of 5-HT (Lapin \& Oxenkrug, 1969) and lower whole blood (Coppen et al. 1976) and platelet (Le Quan-Bui et al. 1984; Quintana, 1992) 5-HT levels have been found in those with depressive illness. Folate deficiency has also been associated with decreased 5-HT activity (Botez et al. 1979b). Supplementation with folic acid has been known to increase cerebrospinal fluid levels of 5-hydroxyindoleacetic acid in folate-deficient patients with depression (Botez et al. 1982), whilst SAM has been reported 
to possess antidepressant properties (Carney et al. 1983; Reynolds \& Stramentinoli, 1983). Given that folate is critical for SAM formation, and the subsequent role of SAM in relation to mood, folic acid supplementation may help to improve mood.

There is also accumulating evidence that elevated homocysteine, which accrues in response to low folate status, may be detrimental to psychological well-being. Up to $30 \%$ of clinically depressed patients have raised plasma homocysteine levels (Bottiglieri et al. 2000). Homocysteine can inhibit the methylation of biogenic amines and phospholipids in the central nervous system (Bottiglieri et al. 1994) such that depressed patients with elevated levels of homocysteine possess lower levels of 5-HT in cerebrospinal fluid (Botez et al. 1979b).

Together, the evidence argues for a relationship between folate and mood, and between folate, neurotransmitter synthesis and release. Despite previous findings amongst clinically depressed subgroups indicating that folate may impact upon neurochemistry, no studies appear to have looked at mood specifically in response to folic acid in healthy individuals. However, two studies have explored mood in response to multivitamin supplementation (Benton et al. 1995; Bryan et al. 2002). Bryan et al. (2002) examined subjective mood in response to a combined B-vitamin supplement (folic acid, vitamin $\mathrm{B}_{6}$, vitamin $\mathrm{B}_{12}$ ) but found no effect on mood. Benton et al. (1995) also found no evidence for a relationship between folate status and mood in response to a high-dose multivitamin supplement. No research to date has considered the impact specifically of folic acid upon neurotransmitter status and in relation to the mood of healthy individuals. The present placebo-controlled trial therefore sought to determine the impact of folic acid supplementation upon subjective mood and a biochemical marker of mood (5-HT) in healthy males.

\section{Materials and methods}

\section{Participants}

Ethical approval was obtained from the Research Ethical Committee of the University of Ulster. Screening and recruitment of participants commenced in February 2002. Exclusion criteria included depression and diseases known to impact upon folate status and/or homocysteine levels, including gastrointestinal disease, haematological disorders and vascular, hepatic or renal disease. Those taking supplements and foods fortified with folic acid or other B-vitamins, or taking medication known to impair folate status, were also excluded. Healthy males $(n 70)$ were recruited amongst university staff/students and from local industries via letters of introduction, poster advertisements and attendance at public/group meetings. Effort was made to recruit from a range of backgrounds to enable the results to be generalised across social groups. All volunteers gave written consent to take part. A fasting blood sample was collected from all potential participants and analysed. Individuals found at screening to be homozygous (TT genotype) for the $677 \mathrm{C} \rightarrow \mathrm{T}$ polymorphism in methylenetetrahydrofolate reductase (TT genotype), which is associated with impaired folate status (Molloy \& Scott, 1997), were also excluded from the study.

\section{Study design and treatment}

The study, a double-blind, placebo-controlled clinical trial design, commenced in June 2002 and was completed by October 2002.
The investigation was carried out as part of a larger intervention study designed to examine the bioavailability of different forms of folate delivered at increasing doses. Participants were randomly allocated to either the folic acid-supplemented ( $n$ 15) or placebo ( $n$ 13) group on the basis of baseline homocysteine values. Those receiving folic acid were initially given $100 \mu \mathrm{g}$ dose for a period of 6 weeks followed by a dose of $200 \mu \mathrm{g}$ for a further 6 weeks. These doses of folic acid are within the physiological range of intake and are likely to be achieved by consumption of foods fortified with folic acid. The intervention period of 6 weeks for each dose of folic acid has been shown to be sufficient in reaching a plateau in serum folate levels (Ward et al. 1997). Supplements were supplied on a weekly basis using $7 \mathrm{~d}$ pill organiser boxes (Carepac, Farringdon, UK). Participants were required to return the pill boxes at the end of each week, including any missed pills so that compliance could be monitored. Abbott SPA, Liscate (MI), Italy supplied the supplements, all of which were identical in appearance, taste and smell to ensure that participants were blind as to their composition.

\section{Blood sampling and analysis}

Fasting blood samples $(12 \mathrm{~h})$ were collected prior to the intervention and at the end of each dose period (every 6 weeks) between 08.00 and 09.00 hours by a qualified nurse/phlebotomist. In total, three blood samples were collected: at baseline (week 0); following the $100 \mu \mathrm{g}$ dose (week 6); following the $200 \mu \mathrm{g}$ dose (week 12; Fig. 1). Blood samples $(19.5 \mathrm{ml})$ were collected by venepuncture, deposited into EDTA-coated pre-evacuated tubes $(1 \times 8 \mathrm{ml}$ and $1 \times 4.5 \mathrm{ml}$ ) and analysed for a full haematological profile, whole blood 5-HT, erythrocyte folate and plasma homocysteine. Samples were also deposited into a separate $7 \mathrm{ml}$ Vacuette Serum-Separator tube (Greiner Labortechnik, Germany) for analysis of serum folate. Post-collection, all blood samples were placed on ice until processing. They were processed and aliquoted within $1-2 \mathrm{~h}$ of collection and then stored at $-70^{\circ} \mathrm{C}$ for batch analysis within 6 months.

Whole blood 5-HT was analysed by means of an HPLC method with fluorescence detection (excitation $270 \mathrm{~nm}$, emission $330 \mathrm{~nm}$ ) as described by Xiao et al. (1998). The blood specimen was thawed on ice and a $400 \mu l$ sample mixed with $400 \mu l$ ice-cold perchloric acid $(0.8 \mathrm{~mol} / \mathrm{l})$ containing $0.1 \mathrm{~mol}$ ascorbic acid/l and 10 mmol EDTA/l. The mixture was then centrifuged in the cold for $10 \mathrm{~min}$ and $20 \mu \mathrm{l}$ of the clear supernatant injected onto a Nucleosil $3 \mu \mathrm{m} \mathrm{C}_{18}$ HPLC column (Phenomenex, Macclesfield, UK). A calibration curve covering $0 \cdot 125-2 \cdot 0 \mu \mathrm{mol} 5-\mathrm{HT} / 1$ was run with every batch of samples. The total $\mathrm{CV}$ for a blood sample containing $0.9 \mu \mathrm{mol} 5-\mathrm{HT} / 1$ was $8.5 \%$ (n 16). Samples were analysed for a full haematological profile using an automated Coulter Counter (Causeway Health and Social Services Trust Laboratories, Coleraine, Northern Ireland). Plasma homocysteine was measured by fluorescence polarisation immunoassay adapted to the Abbott $\operatorname{Imx}{ }^{\circledR}$ Analyzer (Leino, 1999). Serum folate and erythrocyte folate were assessed using the Lactobacillus casei microbiological assay (Molloy \& Scott, 1997). Whole blood samples were analysed for 5-HT (Xiao et al. 1998) at the Alcohol Laboratory of the Karolinska Hospital, Stockholm, Sweden.

\section{Subjective mood assessment}

The Positive and Negative Affect Schedule ('PANAS scale') was used to determine mood. The PANAS, devised by Watson et al. 


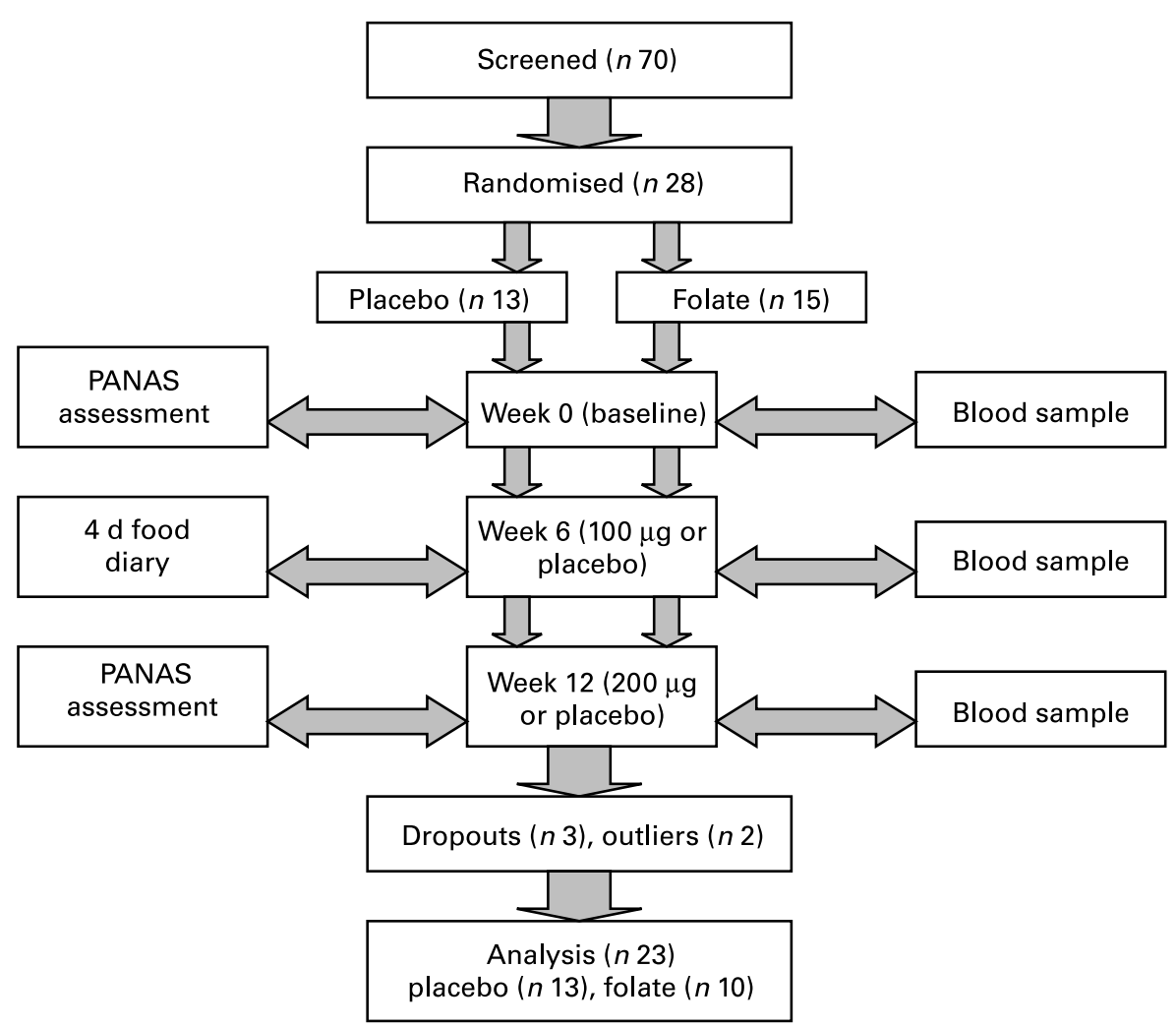

Fig. 1. Scheme of the clinical intervention. PANAS, Positive and Negative Affect Schedule.

(1988), is a validated scale with which to measure subjective mood. For the purpose of the PANAS, mood has been defined as a 'transient episode of feeling or affect' (Watson, 2000, p. 4). This self-administered scale measures two broad distinct dimensions of 'affect' (mood). Positive affect refers to the extent to which a person feels enthusiastic, active or alert. Low positive affect implies sluggishness. Negative affect refers to a general dimension of subjective distress, encompassing a variety of aversive mood states such as anger, contempt, disgust, fear and nervousness. Low negative affect implies calmness and serenity (Watson et al. 1988). Mood variability, the extent to which an individual's mood deviates from average levels to extremes (mood swings), can be considered a stable individual characteristic (McConville \& Cooper, 1992). Mood swings are believed to be important in the aetiology of non-clinical depressed states (Costello et al. 1991; McConville \& Cooper, 1996). It is therefore important to take variability into account in assessing subjective mood. The PANAS has been used previously in conjunction with other common measures of distress and psychopathology. Watson \& Clarke (1997) have shown validity of a subset of the PANAS-X scales with the previously devised Profile of Mood States, whilst it has also been shown to correlate with the Beck Depression Inventory (Watson et al. 1988). With regard to biological markers, the PANAS has been found to correlate with prolactin (Zald \& Depue, 2001) and brain choline uptake (Jung et al. 2002). Taken together, associations between the PANAS, other mood scales and biomarkers indicate its value as a clinical tool for the measurement of mood.

All subjective mood measurements corresponded to blood sampling at both baseline and post-intervention. Mood was assessed using the PANAS over a period of 2 weeks, 1 week prior to the intervention (week 0 ; at baseline) and 1 week at the end of intervention (week 11-12). The PANAS was completed twice daily (at 6 and $12 \mathrm{~h}$ after waking), making a total of fourteen subjective mood assessments per week. The PANAS comprises a list of adjectives, ten of which relate to positive mood and ten to negative mood. Responses are made according to a five-point Likert scale $(1=$ very slightly to $5=$ extremely $)$. Individuals were prompted by the researcher to complete the questionnaire by mobile phone text message, telephone call or email.

\section{Dietary assessment and dietary considerations}

Participants were also asked to complete a $4 \mathrm{~d}$ food diary during the intervention (week 6), over two weekdays and two weekend days. Portion sizes were estimated using household measurements and subsequently quantified using approved food portion sizes (Crawley, 1988). Energy and nutrient (including folate) intakes were computed using the WISP program (Wisp for Windows, Version 1.28; Tinuviel Software, Warrington, UK). Participants were asked to refrain from eating foods reported to alter whole blood 5-HT levels (bananas and walnuts) for the duration of the intervention (Kema et al. 1992; Xiao et al. 1998).

\section{Statistical analysis}

Statistical analysis was performed using the Statistical Package for Social Sciences, Version 11.0 (SPSS Inc., Chicago, IL, USA). Analysis of covariance was employed to determine differences between the folic acid-supplemented and placebo groups in relation to subjective and biochemical markers of mood 
post-intervention taking baseline measures of mood into account as a covariate. Subjective mood was inferred from the mean of each individual's score for positive and negative affect (PANAS) and variability in subjective mood from the mean SD of each individual's positive and negative affect (PANAS) score. Each individual was assigned a score summarising responses across adjectives representing positive mood and negative mood and variation therein for each occasion on which assessments were made. No differences were observed at baseline in mood ratings by time of day or day of week. Mean scores for positive and negative mood and mood variability were therefore summed over time of day and day of week for each individual and entered into the analysis.

Outcome variables found to have a skewed distribution, including measurements of affect, baseline homocysteine, serum and erythrocyte folate, were log-transformed to achieve approximate 'normality'. Estimated marginal means were then entered into analysis of covariance to determine differences over time and by treatment. The effect estimates and associated CI for log-transformed outcome measurements were subsequently back-transformed. The results presented here therefore represent ratios of group geometric means. The arithmetic mean was used to describe baseline blood biochemical measurements and baseline affect. Analysis of covariance was carried out separately for each of the four measurements of affect (positive affect and positive affect variability, negative affect and negative affect variability). In each case, post-intervention measures of affect were analysed, conditional upon the corresponding baseline measures. Post-intervention affect was therefore the 'dependent variable', baseline affect the 'covariate' and treatment group the 'fixed factor'.

\section{Results}

Sample description

A 'per-protocol' analysis was carried out whereby volunteers who did not complete the study $(n 5)$ were excluded from the entire analysis. The resulting sample comprised males ( $n$ 23) assigned to either one of two treatment groups: folic acid $(n 10)$ or placebo ( $n$ 13). Participants ranged in age from 21 to 39 years (mean age 32 years). With regard to social class, $26 \%$ were managerial/professionals, $13 \%$ were non-manual skilled workers, $48 \%$ were manually skilled workers and $13 \%$ were unskilled (National Statistics Socio-Economic Classification, 2002). No differences were observed between the placebo and treatment groups in terms of age or social class.

\section{Baseline results}

No differences were observed in erythrocyte or serum folate, homocysteine, 5-HT (Table 1) or subjective mood (affect; Table 2) between the treatment and placebo group at baseline. Neither serum/erythrocyte folate nor homocysteine was associated with subjective mood prior to the intervention.

\section{Response to folic acid intervention}

Treatment resulted in an increase in erythrocyte folate concentration in response to $200 \mu \mathrm{g} / \mathrm{d}$ folic acid $(P=0.003)$ and an increase in serum folate levels in response to both doses of folic acid $(100 \mu \mathrm{g} / \mathrm{d}, P=0.043 ; 200 \mu \mathrm{g} / \mathrm{d}, P=0.024$; Table 3$)$. Correspondingly, homocysteine decreased as a result of folic acid treatment at both the $100 \mu \mathrm{g} \quad(P=0.032)$ and $200 \mu \mathrm{g}$

Table 1. Baseline blood measurements by treatment group

(Arithmetic mean values with their standard deviation)

\begin{tabular}{|c|c|c|c|c|c|}
\hline \multirow[b]{2}{*}{ Biochemical measure } & \multirow[b]{2}{*}{ Reference range* } & \multicolumn{2}{|c|}{ Folate } & \multicolumn{2}{|c|}{ Placebo } \\
\hline & & Mean & SD & Mean & SD \\
\hline Erythrocyte folate $(\mathrm{nmol} / \mathrm{l})$ & $340-2266$ & $969 \cdot 3$ & $525 \cdot 9$ & $1034 \cdot 5$ & 323.5 \\
\hline Serum folate $(\mathrm{nmol} / \mathrm{l})$ & $6 \cdot 80-45 \cdot 3$ & 9.9 & $7 \cdot 3$ & $9 \cdot 2$ & $5 \cdot 6$ \\
\hline Homocysteine $(\mu \mathrm{mol} / \mathrm{l})$ & $\leq 15$ & 11.4 & 3.4 & $10 \cdot 5$ & $2 \cdot 8$ \\
\hline $5-\mathrm{HT}(\mu \mathrm{mol} / \mathrm{l})$ & $0.5-2 \cdot 0$ & $1 \cdot 0$ & 0.6 & 0.8 & 0.2 \\
\hline
\end{tabular}

5-HT, 5-hydroxytryptamine.

${ }^{*}$ Reference ranges shown are in accordance with the Vitamin Research Laboratory, Trinity College, Dublin for erythrocyte/serum folate; Kang et al. (1992) for homocysteine; and Xiao et al. (1998) for 5-HT.

Table 2. Baseline and post-intervention affect by treatment (Arithmetic mean values with their standard deviation)

\begin{tabular}{|c|c|c|c|c|c|c|c|c|}
\hline \multirow[b]{3}{*}{ Affect } & \multicolumn{4}{|c|}{ Baseline } & \multicolumn{4}{|c|}{ Post-intervention } \\
\hline & \multicolumn{2}{|c|}{ Placebo } & \multicolumn{2}{|c|}{ Folate } & \multicolumn{2}{|c|}{ Placebo } & \multicolumn{2}{|c|}{ Folate } \\
\hline & Mean & SD & Mean & SD & Mean & SD & Mean & SD \\
\hline Positive affect (PA) & 28.47 & $6 \cdot 90$ & $24 \cdot 03$ & 4.89 & $28 \cdot 34$ & $8 \cdot 22$ & 23.53 & 4.05 \\
\hline PA variation & $9 \cdot 84$ & 2.05 & $9 \cdot 21$ & $2 \cdot 11$ & $9 \cdot 41$ & 1.85 & 8.82 & 2.43 \\
\hline Negative affect (NA) & $12 \cdot 78$ & 1.95 & $13 \cdot 35$ & 3.97 & $12 \cdot 7$ & 3.00 & 13.41 & 3.20 \\
\hline NA variation & $5 \cdot 88$ & 2.51 & $5 \cdot 86$ & $2 \cdot 55$ & 5.03 & $2 \cdot 60$ & $6 \cdot 65$ & 3.72 \\
\hline Total affect variation & $6 \cdot 85$ & 1.32 & $6 \cdot 44$ & 1.85 & $6 \cdot 15$ & 1.54 & $6 \cdot 63$ & 1.93 \\
\hline
\end{tabular}


Table 3. Post-intervention blood measurements

\begin{tabular}{|c|c|c|c|c|c|c|}
\hline & \multicolumn{6}{|c|}{ Post-intervention difference } \\
\hline & \multicolumn{3}{|c|}{$100 \mu \mathrm{g}$ dose } & \multicolumn{3}{|c|}{$200 \mu \mathrm{g}$ dose } \\
\hline & Mean difference & $95 \% \mathrm{Cl}$ & $P$ value & Mean difference & $95 \% \mathrm{Cl}$ & $P$ value \\
\hline Erythrocyte folate* $(\mathrm{nmol} / \mathrm{l})$ & 1.07 & $0.94,1.21$ & 0.298 & $1 \cdot 31$ & $1.11,1.55$ & 0.003 \\
\hline Serum folate* $(\mathrm{nmol} / \mathrm{l})$ & 1.45 & $1.01,2.08$ & 0.043 & 1.44 & $1.05,1.95$ & 0.024 \\
\hline Homocysteine ( $\mu \mathrm{mol} / \mathrm{l})$ & -1.45 & $-2.76,-0.14$ & 0.032 & -1.48 & $-2.64,-0.32$ & 0.015 \\
\hline 5-HT $(\mu \mathrm{mol} / \mathrm{l})$ & 0.04 & $-0.21,0.30$ & 0.732 & 0.03 & $-0 . .23,0.29$ & 0.816 \\
\hline
\end{tabular}

5-HT, 5-hydroxytryptamine.

* Erythrocyte and serum folate were back-transformed; therefore the ratio values are shown instead of the mean difference.

$(P=0 \cdot 015)$ folic acid dose (Table 3$)$. These results indicate compliance with the intervention. Whole blood 5-HT levels did not differ between the placebo or treatment group post-intervention. Nor were any differences observed in relation to subjective mood (positive affect, negative affect or variability in positive or negative affect) between the treatment and placebo groups postintervention (Table 4). The CI indicate no more than a 10$15 \%$ difference between groups. Positive and negative affect and positive and negative affect variability were unrelated to serum or erythrocyte folate and homocysteine post-intervention.

\section{Discussion}

It was hypothesised that mood would alter in response to folic acid supplementation and that this would be reflected in both measures of subjective mood (PANAS scores) and 5-HT in whole blood, a biochemical marker of mood. As expected, erythrocyte and serum folate increased while homocysteine decreased post-intervention in the folate-supplemented but not the placebo group, indicating good efficacy and compliance within the treatment group, and lending validity to the reported findings. Supplementation with folic acid, however, did not appear to alter either subjective mood (affect) or serotonin in this sample of healthy males. Nor did folic acid appear to be detrimental to mood. Mood instability has been associated with depressive states (McConville \& Cooper, 1996). There was no evidence for any relationship between mood variability and post-intervention folic acid blood levels in these folate-replete individuals.

Few studies appear to have examined the effect of folate supplementation upon mood in healthy individuals and no previous research appears to have considered 5-HT in response to folic acid supplementation. Those that exist have supplemented the clinically depressed, often older patients usually deficient in folate, and without employing placebo controls (Botez et al. 1979a; Guaraldi et al. 1993). In some instances, intervention with folic acid has been in addition to antidepressant treatment (Coppen et al. 1986; Godfrey et al. 1990; Coppen \& Bailey,

Table 4. Estimated ratio between geometric means postintervention

\begin{tabular}{lccc}
\hline Affect & Ratio & $95 \% \mathrm{Cl}$ & $P$ value \\
\hline Positive affect (PA) & 1.00 & $0.88,1.12$ & 0.9 \\
PA variability & 0.97 & $0.83,1.13$ & 0.7 \\
Negative affect (NA) & 1.00 & $0.86,1.17$ & 0.9 \\
NA variability & 1.14 & $0.73,1.78$ & 0.5 \\
\hline
\end{tabular}

2000). Only two previous studies appear to have examined the impact of folate status upon mood in healthy volunteers. Both of these studies intervened using a combined multivitamin supplement, making it difficult to draw conclusions specifically relating to folic acid (Benton et al. 1995; Bryan et al. 2002). Nevertheless, consistent with our findings, neither of these previous studies observed any change in the mood of healthy individuals as measured by the Profile of Mood States Questionnaire (McNair et al. 1971). Given that the Profile of Mood States and the PANAS have been found to have good correspondence (Watson \& Clarke, 1997), together these results suggest that folic acid, in physiological doses, over a limited period of time improves folate status but does not benefit mood in healthy individuals. There is some evidence to imply that high levels of homocysteine are associated with depression (Bottiglieri et al. 2000). Homocysteine levels greater than $15 \mu \mathrm{mol} / \mathrm{l}$ have been shown to contribute towards increased depressive symptoms and lowered mood in older people (Jensen et al. 1998). Lowering of homocysteine in response to folic acid could therefore be expected to contribute towards improved mood. The observed decrease in homocysteine in response to folic acid supplementation in the current study, however, did not produce any apparent change in mood. That said, baseline plasma homocysteine concentrations of the participants in the current study ranged between 7 and $14 \mu \mathrm{mol} / \mathrm{l}$ and none had levels above $15 \mu \mathrm{mol} / \mathrm{l}$. That baseline homocysteine levels were not particularly elevated and were well below the $15 \mu \mathrm{mol} / \mathrm{l}$ threshold often used to indicate hyperhomocysteinaemia (Kang et al. 1992) could also explain apparent discrepancies between our findings and those in some of the previous studies (Jensen et al. 1998; Bottiglieri et al. 2000). However, our results agree with the findings of Morris et al. (2003) and Alpert \& Fava (1997). They also failed to detect an association between homocysteine and mood.

A possible reason for the lack of improvement in either subjective or biochemical measures of mood in response to folic acid is that a curvilinear relationship may exist between folate dose and antidepressant effect (Alpert \& Fava, 1997). Folic acid supplementation may therefore only enhance the mood of those with depression and/or folate deficiency. Previous research reporting a mood-related response to folic acid supplementation has tended to focus upon those who are either folate-deficient or experiencing poor mental health (Carney, 1967; Reynolds et al. 1970; Botez et al. 1979a; Guaraldi et al. 1993). In the current investigation no change in mood was evident. This may have been as a consequence of normal folate status and psychological well-being at baseline. In accordance with this view, the only evidence relating to the impact of folic acid on healthy individuals 
(Benton et al. 1995; Bryan et al. 2002) found no specific response to a multivitamin supplement containing folic acid, findings that are consistent with our results.

Alternative explanations for the current findings include the possibility that the period of intervention may not have been long enough to observe a response in mood, or that the folic acid dose may have been insufficient. Previous placebo-controlled trials that have reported improvement in mood have supplemented with extremely large doses of folic acid $(15 \mathrm{mg} / \mathrm{d})$ over a period of 4 months (Botez et al. 1979a) or methyltetrahydrofolate $(50 \mathrm{mg} / \mathrm{d})$ over 6 weeks (Guaraldi et al. 1993) in folate-deficient clinically depressed patients. That the current investigation exclusively sampled males could also explain the null findings. Benton et al. (1995) previously found that females, but not males, experienced improvement in mood in response to a combined vitamin supplement, which included folic acid. Males also tend to have lower levels of whole blood 5-HT than females (Coppen et al. 1976). Males and females could therefore be expected to respond differently in terms of psychological outcomes to nutrient interventions. Further research is required to explore sex differences in psychological response to folic acid.

The small study sample employed may limit the conclusions that can be drawn from these results. The study had $80 \%$ power to detect a between-group difference larger than 1.2 of the within-group SD. Given the SD of the PANAS scores at baseline, the smallest difference that can be ruled out with $80 \%$ power is one of three scale points in the case of negative mood and one of six scale points in the case of positive mood. The likelihood of making a type II error, that is, of not detecting a difference when one exists, is therefore considerable. However, there are difficulties in determining what constitutes a clinically meaningful difference in the mood scores as so little clinical research to date has used the PANAS scale. Researchers who have previously used the PANAS alongside biomarkers of mood have used similar small sample sizes. For example, Jung et al. (2002) assessed the relationship between the PANAS and brain choline levels ( $n$ 27) and found choline to be inversely related to positive affect. Zald \& Depue (2001) used the PANAS alongside measures of prolactin, a marker of serotonergic functioning ( $n$ 34), and found prolactin to be inversely associated with both positive and negative affect. That notable differences have been observed in relation to other markers of mood such as choline and prolactin using the PANAS by means of similar sample sizes suggests that neither the psychometric instrument employed nor the sample size is likely to have been responsible for the null findings resulting from the present study.

Another factor, which may have influenced the current results, is seasonal variation. Seasonal variation can influence both 5-HT status and mood. Platelet 5-HT concentrations ( $\geq 98 \%$ of whole blood 5-HT is in the platelets) have been known to show seasonal variations in healthy males (Sarrias et al. 1989; Brewerton et al. 1993). Minimum values of whole blood 5-HT are typically observed during autumn (October-November) and maximum levels during spring (May-June) and summer (August). Positive affect tends to be higher during spring/summer (Watson, 2000). Negative mood levels appear highest in August-November and lowest in either February-May or June-July (Harris \& Dawson-Hughes, 1993). Positive mood would probably have been optimal at the time the study was carried out (JuneOctober). This may explain why positive mood did not appear to respond to folic acid supplementation. That no variation in mood was observed in the placebo group over the course of the research, however, implies that seasonal factors did not confound the results. Baseline subjective mood scores were also within normal ranges observed in similar populations (Watson \& Clarke, 1994; McConville \& Cooper, 1999). Future research should nevertheless seek to intervene with folic acid during the period from February through to April.

The current study, the first to our knowledge to have specifically investigated the effect of folic acid on mood in nondepressed individuals, differed from previous trials by considering both subjective self-reported and biochemical measures of mood.

The results suggest that short-term supplementation with folic acid does not influence either subjective or biochemical markers of mood (5-HT), when given at physiological doses to healthy folate-replete male volunteers with normal mood. Future research would merit the use of a larger sample comprising both males and females, and should consider increased dose and duration, and the impact of seasonal variation.

\section{Acknowledgements}

We gratefully acknowledge the contribution of Professor John Scott and Dr Anne Molloy, Trinity College Dublin, who carried out the homocysteine and folate analysis. Funding for these measurements and intervention costs was provided as part of the EU project 'Nutraceuticals for a Healthier Life: $n$-3 polyunsaturated fatty acids and 5-methyltetrahydrofolate' (NUHEAL; QLRT-1999-00 888).

\section{References}

Agnoli A, Ruggieri S \& Cerrone GG (1977) The dopamine hypotheses of depression: results of treatment with dopaminergic drugs. In Depressive Disorders, pp. 447-458 [S Grattini, editor]. Stuttgart: FK Schatauer Verlag.

Alpert JE \& Fava M (1997) Nutrition and depression: the role of folate. Nutr Rev 55, 145-149.

Benton D, Haller J \& Fordy J (1995) Vitamin supplementation for 1 year improves mood. Neuropsychobiology 32, 98-105.

Botez MI, Botez T, Levielle J, Bielmann T \& Cadotte M (1979a) Neuropsychological correlates of folic acid deficiency: facts and hypotheses. In Folic Acid in Neurology, Psychiatry, and Internal Medicine, pp. 425-461 [MI Botez and EH Reynolds, editors]. New York: Raven Press.

Botez MI, Young SN, Bachevalier J \& Gauthier S (1979b) Folate deficiency and decreased brain 5-hydroxytryptamine synthesis in man and rat. Nature 278, 182-183.

Botez MI, Young SN, Bachevalier J \& Gauthier S (1982) Effect of folic acid and vitamin $\mathrm{B}_{12}$ deficiencies on 5-hydroxyindoleacetic acid in human cerebrospinal fluid. Am Neurol Assoc 12, 479-484.

Bottiglieri T, Hyland K \& Reynolds EH (1994) The clinical potential of ademethionine ( $S$-adenosylmethionine) in neurological disorders. Drugs 48, 137-152.

Bottiglieri T, Laundy M, Crellin R \& Toone BK (2000) Homocysteine, folate, methylation, and monoamine metabolism in depression. $J$ Neurol Neurosurg Psychiatry 69, 228-234.

Brattstrom L, Israellson B, Jeppson J \& Hultberg B (1998) Folic acid - an innocuous means to reduce plasma homocysteine. Scand J Clin Lab Invest 48, 215-221.

Brewerton TD, Flament MF, Rapoport JL \& Murphy DL (1993) Seasonal effects on platelet $5 \mathrm{HT}$ content in patients with OCD and controls. Arch Gen Psychiatry 50, 409. 
Bryan J, Calvaresi E \& Hughes D (2002) Short-term folate, vitamin B-12 or vitamin B-6 supplementation slightly affects memory performance but not mood in women of various ages. J Nutr 132, 1345-1356.

Cantoni GL (1953) S-Adenosylmethionine: a new intermediate formed enzymatically from L-methionine and adenosine triphosphate. $J$ Biol Chem 204, 403-416.

Carney MWP (1967) Serum folate values in 423 psychiatric patients. $\mathrm{Br}$ Med J 4, 512.

Carney MWP, Martin R, Bottiglieri T, Reynolds EH, Nissenbaum H, Toone BK \& Sheffield BN (1983) The switch mechanism in affective illness and $S$-adenosylmethionine. Lancet 1, 820-821.

Coppen A \& Bailey J (2000) Enhancement of the antidepressant action of fluoxetine by folic acid: a randomised, placebo controlled trial. J Affect Disord 60, 121-130.

Coppen A, Turner P, Rowsell AR \& Padgham C (1976) 5-Hydroxytryptamine (5-HT) in the whole-blood of patients with depressive illness. Postgrad Med J 52, 156-158.

Coppen A, Chaudhry S \& Swade C (1986) Folic acid enhances lithium prophylaxis. J Affect Disord 10, 9-13.

Costello J, Benjamin R, Angold A \& Silver D (1991) Mood variability in adolescents. A study of depressed, nondepressed and comorbid patients. J Affect Disord 23, 199-212.

Crawley H (1988) Food Portion Sizes, 3rd ed. London: HMSO.

Fava M, Borus JS, Alpert JE, Nierenberg AA, Rosenbaum JF \& Bottiglieri $\mathrm{T}$ (1997) Folate, vitamin $\mathrm{B}_{12}$, and homocysteine in major depressive disorder. Am J Psychiatry 154, 426-428.

Ghadirian AM, Anath J \& Engelsmann F (1980) Folic acid deficiency in depression. Psychosomatics 21, 926-929.

Godfrey PSA, Toone BK, Carney MWP, Flynn TG, Bottiglieri T, Laundy M, Chanarin I \& Reynolds EH (1990) Enhancement of recovery from psychiatric illness by methylfolate. Lancet 336, 392-395.

Guaraldi G, Fava M, Mazzi F \& LaGreca P (1993) An open trial of methyltetrahydrofolate (MTHF) in elderly depressed patients. Ann Clin Psychiatry 5, 101-106.

Harris S \& Dawson-Hughes B (1993) Seasonal mood changes in 250 normal women. Psychiatry Res 49, 77-87.

Herbert V (1962) Experimental nutritional folate deficiency in man. Trans Assoc Am Physicians 75, 307-320.

Jensen E, Dehlin O, Erfurth EM, Hagberg B, Samuelsson B, Svensson T \& Hultberg B (1998) Plasma homocysteine in 80-year-olds: relationships to medical, psychological and social variables. Arch Gerontol Geriatr 26, 215-226.

Jung RE, Yeo RA, Love TM, Petropoulos H, Sibbitt TL \& Brooks WM (2002) Biochemical markers of mood: a proton magnetic resonance spectroscopy study of normal human brain. Biol Psychiatry 51, 224-229.

Kang SS, Wong PWK \& Malinow MR (1992) Hyperhomocyst(e)inemia as a risk factor for occlusive vascular disease. Annu Rev Nutr 12, 279-298

Kema IP, Schellings AMJ, Melmorg G, Hoppenbrouwers CJM \& Muskiet FAJ (1992) Influence of a serotonin- and dopamine-rich diet on platelet serotonin content and urinary excretion of biogenic amines and their metabolites. Clin Chem 38, 1730-1736.

Lapin IP \& Oxenkrug GF (1969) Intensification of the central serotonergic processes as a possible determinant of the thymoleptic effect. Lancet $\mathbf{1}$, $132-136$.
Leino A (1999) Fully automated measurement of total homocysteine in plasma and serum on the Abbott IMx Analyzer. Clin Chem 45, 569-571.

Le Quan-Bui KH, Plaisant O, Leboyer M, Gay C, Kamal L, Devynck M \& Meyer P (1984) Reduced platelet serotonin in depression. Psychiatry Res 13, 129-139.

McConville C \& Cooper C (1992) Mood variability and personality. Pers Individ Dif 13, 1213-1221.

McConville C \& Cooper C (1996) Mood variability and the intensity of depressive states. Curr Psychol 14, 329-338.

McConville C \& Cooper C (1999) Personality correlates of variable moods. Pers Individ Dif 26, 65-78.

McNair DM, Lorr M \& Dropplemann F (1971) Profile of Mood States. San Diego, CA: Educational and Industrial Testing Service.

Molloy AM \& Scott JM (1997) Microbiological assay for serum, plasma, and red cell folate using cryopreserved, microtiter plate method. Methods Enzymol 281, 43-53.

Morris MS, Fava M, Jacques PF \& Selhub J (2003) Depression and folate status in the US population. Psychother Psychosom 72, 80-87.

National Statistics Socio-Economic Classification (2002) National Statistics Socio-Economic Classification User Manual. London: HMSO, Office for National Statistics.

Ordonez LA \& Wurtman RJ (1974) Folic acid deficiency and methyl group metabolism in rat brain: effects of L-Dopa. Arch Biochem Biophys 160, 372-376.

Quintana J (1992) Platelet serotonin and plasma tryptophan in endogenous depression. Clinical, therapeutic, and biological correlations. Affect Disord 24, 55-62.

Reynolds EH \& Carney MWP (1984) Methylation and mood. Lancet 28, 196-198.

Reynolds EH \& Stramentinoli G (1983) S-Adenosylmethionine and affective disorder. Psychol Med 13, 705-710.

Reynolds EH, Preece JM, Bailey J \& Coppen A (1970) Folate deficiency in depressive illness. Br J Psychiatry 117, 287-292.

Sarrias MJ, Artigas M, Martinez A \& Gelpi E (1989) Seasonal changes of plasma serotonin and related parameters: correlations with environmental measures. Biol Psychiatry 26, 695-706.

Ward M, McNulty H, McPartlin J, Strain JJ, Weir DG \& Scott JM (1997) Plasma homocysteine, a risk factor for cardiovascular disease, is lowered by physiological doses of folic acid. Q J Med 90, 519-524.

Watson D (2000) Mood and Temperament. New York: The Guilford Press.

Watson D \& Clark LA (1997) Measurement and mismeasurement of mood; recurrent and emergent issues. J Pers Assess 66, 267-296.

Watson D \& Clark LA (1994) The PANAS-X Manual for the Positive and Negative Affect Schedule - Expanded Form. Ames, IA: University of Iowa.

Watson D, Clark LA \& Tellegen A (1988) Development and validation of brief measures of positive and negative affect: the PANAS scales. $J$ Pers Soc Psychol 54, 1063-1070.

Wesson VA, Levitt AJ \& Joffe RT (1994) Change in folate status with antidepressant treatment. Psychiatry Res 53, 313-322.

Xiao R, Beck O \& Hjemdahl P (1998) On the accurate measurement of serotonin in whole blood. Scand J Clin Lab Invest 58, 505-510.

Zald DH \& Depue RA (2001) Serotonergic functioning correlates with positive and negative affect in psychiatrically healthy males. Pers Individ Dif 30, 71-86. 